\title{
Confiabilidad y validez del cuestionario de trastornos de sueño BEARS en niños y adolescentes escolares de Bogotá, D.C., Colombia: Estudio FUPRECOL Reliability and Validity of the BEARS Sleep Disorder Questionnaire in Children and Adolescent Students from Bogotá, D.C., Colombia: FUPRECOL Study
}

Robinson Ramírez-Vélez, Libardo Huertas-Zamora, Jorge Enrique Correa-Bautista, Edgar Giovanni Cárdenas-Calderón Universidad del Rosario, Bogotá(Colombia)

Resumen. El sueño juega un papel fundamental en muchas esferas del desarrollo de los infantes en etapa escolar. El objetivo de este estudio fue determinar la confiabilidad y validez de contenido del cuestionario BEARS en niños y adolescentes de 9 a 17 años. La muestra estuvo compuesta por 8.862 niños y adolescentes pertenecientes a 24 instituciones educativas oficiales de Bogotá, Colombia. Se aplicó el cuestionario BEARS, de manera auto-diligenciada por formulario estructurado. Una sub-muestra de 246 participantes se utilizó para los análisis de fiabilidad. La consistencia del instrumento se analizó mediante el coeficiente alfa de Cronbach, la validez de constructo a través del análisis factorial exploratorio (AFE), y la fiabilidad mediante el coeficiente Kappa ponderado. Los resultados mostraron adecuada consistencia (Cronbach global de 0,732; rango 0,706 a 0,769) y moderada fiabilidad (kappa de 0,665; rango 0,378 a 0,629). El AFE determinó un solo factor (ítem 1: problemas a la hora de dormir), explicaba el $61,4 \%$ de la varianza, agrupando dos interacciones $\div 2 / g l=2690,817 ; p=0,001$. El cuestionario BEARS mostró una adecuada consistencia interna, fiabilidad y validez. A partir de estos resultados, se recomienda este instrumento principalmente en el contexto de atención primaria, para el estudio y cribado de las alteraciones del sueño en población escolarizada de Bogotá, Colombia.

Palabras clave: Trastornos de sueño, Cuestionarios, Psicometría, Confiablidad, Validación.

\begin{abstract}
Sleep plays a vital role in good health and well-being in school-age children. The aim of this study was to determine the reliability and content validity of the BEARS questionnaire in children and adolescents from 9 to 17 years of age. The sample comprised 8,862 children and adolescents belonging to 24 official education institutions from Bogotá, Colombia. The BEARS questionnaire was applied through self-completion manner, with a structured form. A sub-sample of 246 participants was used for reliability analyses. Consistency of the instrument was analyzed through Cronbach's alpha coefficient, construct validity through exploratory factor analysis (EFA), and reliability through the weighted Kappa coefficient. Results showed adequate consistency (global Cronbach of 0.732; range 0.706 to 0.769) and moderate reliability (Kappa of 0.665 ; range 0.706 to 0.769 ). The EFA determined a single factor (item 1: problems when going to sleep), which explained $61.4 \%$ of the variance, grouping two interactions $\div / \mathrm{gl}=$ 2690.817; $p=0.001$. The BEARS questionnaire showed adequate internal consistency, reliability, and validity. From these results, this instrument is recommended principally within the context of primary care for the study and screening of sleep alterations in school-aged populations from Bogotá, Colombia.
\end{abstract}

Key words: sleep disorders, questionnaires, psychometrics, reliability, validation.

\section{Introducción}

El sueño juega un papel fundamental en muchas esferas del desarrollo de los niños y adolescentes. Previamente, se ha descirto que los trastornos de sueño (TS) son alteraciones relacionadas con la calidad o la cantidad del sueño; e incluyen dificultades para conciliarlo o mantenerse dormido/despierto durante momentos de actividad (Mindell et al., 2003). Desde etapas tempranas de la vida, los TS conllevan a problemas relacionados con la conducta, el rendimiento escolar, la salud metabólica y la condición física (Miró et al., 2005; García-Jiménez et al., 2004; Salsberg et al., 2015).

Por ejemplo, Alamian et al. (2016) describieron que estudiantes americanos de sexto grado que acusaron alteraciones del sueño, tenían mayor oportunidad de categorizarse en sobrepeso (OR 1,68; IC95\% 1,11-1,25) que los estudiantes con peso saludable. Similar resultado fue reportado por Chaput et al. (2011) en una muestra de 550 niños canadienses de nueve años con sobrepeso u obesidad, en quienes se encontró una relación entre dormir menos de 10 horas y exceso de peso por adiposidad central (OR 2,08; IC95\% 1,16-3,67), hallazgo confirmado por Kong et al. (2011) en niños de Hong Kong, por Choi et al, en Corea del Sur (2008) y por Garaulet et al. (2011) en adolescentes europeos.

En esta misma línea, se ha descrito una relación entre desórdenes del sueño y rendimiento académico (Martins et al. 2014). Gruber et al. (2014) hallaron que en niños canadienses que tenían una mejor calidad del sueño, presentaban mayores resultados en las asignaturas de matemáticas y en habilidades para un segundo idioma. Esta misma relación, fue reportada por Anderson et al. (2009) en una muestra de jóvenes americanos entre un mejor puntaje en las funciones ejecutivas y adecuados patrones del sueño.

De otro lado, se resalta que la prevalencia de los TS en niños y

Fecha recepción: 01-06-17. Fecha de aceptación: 31-10-17 Robinson Ramírez-Vélez robinson.ramirez@urosario.edu.co adolescentes reportados en diferentes estudios, oscilan entre un 12,0 y 61,4\% (Chaput et al., 2011; Ipsiroglu et al., 2002; Paavonen et al., 2000; Liu et al., 2000; Owens \& Dalzell 2005; Ravikiran et al., 2011). Por ejemplo, García-Jiménez et al. (2004) encontraron en adolescentes españoles que el 52,8\% de los encuestados acudían a clases con un tiempo menor a ocho horas de sueño, y de ellos, el 17\% mostraba síntomas de somnolencia diurna excesiva. Garaulet et al. (2011) hallaron que alrededor de uno de cada tres adolescentes europeos entre 12,5 y 17,5 años, no cumplían con las recomendaciones mínimas de sueño (> 8 horas) en esta franja de edad. En este sentido, el estudio de los TS, tienen un especial interés como problema de salud pública (Miró et al., 2005).

Para dterminar la existencia de TS en población infantil y adolescente, se han desarrollado varias escalas entre las que se encuentran el Sleep Impairment Index (Smith \& Trinder et al., 2001), la cual evalúa la percepción del sueño en relación con el desempeño diurno, el SleepWake Activity Inventory (Smith \& Trinder et al., 2001), que valora, la somnolencia, el Sleep Disorders Questionnaire (Smith \& Trinder et al., 2001), que indaga por el sueño fisiológico y sus trastornos relacionados, el School Sleep Habits Survey (Giannotti et al., 2002), que estima hábitos de sueño, somnolencia diurna, asistencia escolar, aspectos emocionales y consumo de sustancias que alteran el mismo; y el Post-Sleep Inventory (Webb et al., 1976) que mide aspectos de calidad antes, durante y después del descanso.

En el ámbito de la atención primaria en salud, la escala más usada es la Child Sleep Habits Questionnaire (CSHQ) (Waumans et al., 2010; Owens et al., 2000; Lucas-de la Cruz et al., 2016; Fallahzadeh et al., 2015; Li et al., 2015), la cual consta de 22 ítems, y una version de cribado, descrita por Owens et al. (2005), denominada escala BEARS

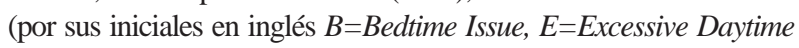
Sleepiness, $A=$ Night Awakenings, $R=$ Regularity anda Duration of Sleep, $S=$ Snoring), que indaga con cinco preguntas los TS más comunes en las categorias (en español B= problemas a la hora de dormir, E= somnolencia diurna excesiva, $\mathrm{A}=$ despertares nocturnos, $\mathrm{R}=$ regularidad y duración del sueño y S= ronquido). Esta versión cuenta con validación en 
Castellano en infantes españoles (Aristizábal et al., 2007) pero en el contexto colombiano, en especial en el ámbito escolar no posee estudios de validación.

Como se mencionó anteriormente, la prevalencia de los TS es alta en la población infantil y adolescente, además de la evidencia de la estrecha relación con alteraciones del estado de salud y el rendimiento académico, situación por la cual se hacen necesarias la adaptación y validación de escalas que permitan evaluar los TS en este grupo poblacional. Por ello, ante la ausencia de estudios de validación de cuestionarios de TS en población escolar colombiana, se realizó el presente trabajo acorde con las normas de validación y revisión de estudios instrumentales, con el objetivo de determinar la confiabilidad y validez de contenido del cuestionario BEARS en una muestra de niños y adolescentes escolares de 9 a 17 años, pertenecientes al estudio FUPRECOL.

\section{Metodología}

\section{Diseño}

El presente estudio presenta un diseño instrumental (Ato, LópezGarcía, \& Benavente, 2013).

\section{Población y muestra}

A partir de un análisis secundario del estudio FUPRECOL (Asociación de la Fuerza Prensil con Manifestaciones Tempranas de Riesgo Cardiovascular en Niños y Adolescentes Colombianos, -financiado por Colciencias en el 2014-, n=11.000 escolares), cuya metodología completa ha sido publicada con anterioridad (Ramírez-Vélez et al., 2015), se estudió una sub-muestra de 8.862 participantes en el componente de confiabilidad interna y validez de contenido. El estudio de fiabilidad, se realizó en otra sub-muestra de 246 escolares con 7 días de diferencia. En ambas sub-muestras, los escolares tenían entre 9 y 17 años de edad, pertenecían al nivel socioeconómico medio y bajo, y estaban matriculados en escuelas públicas del Distrito Bogotá. Los datos fueron obtenidos durante Febrero de 2014 y Noviembre de 2015. El muestreo del presente estudio se realizó de manera no aleatoria, por conveniencia, de manera secuencial, seleccionando los escolares según el cumplimiento de los criterios de elegibilidad y su disponibilidad para participar en el estudio (Ramírez-Vélez et al., 2015).

Se excluyeron escolares con discapacidad física, sensorial e intelectual permanente, enfermedades no transmisibles como diabetes tipo $1 \mathrm{o}$ 2, enfermedad cardiovascular, autoinmune o cáncer diagnosticado, estado de gestación, y, en general patologías que no estuviesen relacionadas directamente con la nutrición como errores congénitos del metabolismo y trastornos psiquiátricos (anorexia, bulimia), etc. Esta exclusión se realizó a posteriori. Para evaluar la validez de contenido, era suficiente contar con un mínimo de 670 escolares asumiendo un error tipo I de 0,05 , un poder de 0,80 , un valor del coeficiente de correlación alfa de Cronbach igual a 0,80 para la hipótesis alterna, un valor del coeficiente de correlación alfa de Cronbach igual a 0,70 para la hipótesis nula, para una escala 5 ítems. Este cálculo se realizo en el programa Study-size $2.0 .4_{\circledast}$

\section{Instrumentos de medida}

Antes de las mediciones del estudio, los investigadores y evaluadores encargados del trabajo de campo realizaron diez sesiones teóricas y prácticas, para estandarizar el proceso de evaluación y diligenciamiento de las encuestas. Inicialmente, se contó con el consentimiento informado de las partes implicadas en el proceso (centro educativo, profesores, tutores y padres), y con el asentimiento de los escolares voluntarios. Los cuestionarios se adminstraron en las instalaciones de los colegios, en grupos de 20 a 50 participantes, en las aulas para mantener la privacidad y libertad en la complementación, y con la presencia de, por lo menos, dos investigadores cualificados. Antes del diligenciamiento de los cuestionarios, se informaron las pautas necesarias para su correcto llenado, y se insistió en la necesidad de prestar atención en la lectura de los ítems y en la sinceridad y el anonimato a la hora de responder el cuestionario. El estudio FUPRECOL se llevó a cabo siguiendo las normas deontológicas reconocidas por la Declaración de Helsinki y la resolución 008430 de 1993 del Ministerio de Salud de Colombia, la cual regula la investigación clínica en humanos. Además, el estudio contó con la aprobación del Comité de Investigación en Seres Humanos de la universidad encargada del estudio (UR N CEI-ABN026-000262).

\section{Variables}

Las variables de sexo y edad, fueron tomadas por auto-reporte. Para la evaluación de los TS se usó un cuestionario para detectar los problemas más comunes del sueño en niños y adolescentes (2 a 18 años) del «BEARS Cribado de Trastornos de Sueño» de Owens et al. (2005), y su versión corta al Castellano en el rango de 6 a 18 años de edad (Aristizábal et al., 2007). Los dominios de la calidad del sueño usados en el presente estudio de validación fueron: 1.) problemas de la hora de dormir/dificultad para irse a la cama y quedarse dormido; 2.) excesiva somnolencia diurna; 3.) despertares durante la noche; y 4.) regularidad y duración del sueño. El ítem 5.) ronquido.

\section{Análisis estadístico}

Inicialmente, se llevó a cabo una descripción de las prevalencias de los TS por edad y sexo para identificar diferencias y conocer el problema en cuestión. Posteriormente, se realizó una evaluación de las propiedades psicométricas y se consideró que el valor de alfa fuera superior a 0,70 (Nunnany 1978), en la comparación entre grupos de sujetos. Adicionalmente se calcularon las modificaciones de ese valor al excluir cada uno de los ítems del cuestionario. La fiabilidad de la prueba o reprueba se realizó por medio del coeficiente de kappa ponderado en las variables de carácter ordinal. Se realizaron dos aplicaciones del formato de valoración en una submuestra de 246 individuos; con una diferencia de tiempo entre la primera y la segunda aplicación de 7 días.

Por último, se aplicó un análisis factorial exploratorio (AFE) por medio del método de componentes principales para conocer la validez de contenido. Para esto, se realizó un análisis paralelo a una réplica de la matriz de datos generada aleatoriamente y se tomó el número de factores para retener; este proceso se repitió 250 veces (Owens et al., 2005). Mediante la elaboración de una matriz de correlación se realizó un análisis factorial de componentes principales, con rotación varimax, según el grado de correlación existente entre ellos, a través de la prueba de esfericidad de Bartlett (Pérez-Moreno et al., 2017). Se definió, el determinante de la matriz de correlaciones y la prueba de Kaiser-MeyerOlkin, valores por encima de (0,5). De esta manera, la inclusión de cada ítem en un determinado factor, se realizó si existía un grado de saturación mínimo de 0,4 y un autovalor mayor de 1 (Pérez-Moreno et al., 2017). La cantidad de factores se estipuló sin restricción de estructura y posteriormente mediante la determinación de un número reducido de factores, según los resultados del screen test. Se tomó una significatividad nominal de 0,05 para todos los cálculos y los datos se analizaron con el paquete comercial estadístico SPSS-IMB versión24(Chicago, IL, USA).

\section{Resultados}

Respecto a la prevalencia de muestra total ( $\mathrm{n}=11.000), 8.862$ escolares, cumplieron con todos los criterios de inclusión y tenían los datos completos del cuestionario de trastornos de sueño «BEARS» (tasa de respuesta $80.5 \%$ ). El 57,3\% pertenecían al grupo femenino, con edad media de $12,9 \pm 2,4$. Las mujeres reportaron un (22\%, «problemas a la hora de dormir» frente al (18\%) de los varones ( $\mathrm{p}<0.05)$, al igual que una «excesiva somnolencia diurna» (28\% mujeres, vs. $22 \%$ varones, $\mathrm{p}<0.05$ ). No se observaron diferencias en el dominio de «despertares nocturnos», por grupos (22\% en mujeres y varones). Frente a la «regularidad y duración del sueño», los varones manifestaron mayores valores que las mujeres (32\% vs. 28\%). El ronquido, fue la prevalencia menor en ambos grupos (varones $6 \%$ vs. $1 \%$ de mujeres). La prevalencia de trastornos de sueño agrupada por sexo y para cada edad se presentan en la Tabla 1.

En laTabla2, se muestran el índice de discriminación y el coeficiente de alfa de Cronbach (indicadores de confiabilidad). La consistencia 
interna por factor presentó valores alfa de cronbach en un rango de 0,706 a 0,769 y un alfa de cronbach general de 0,732. La menor varianza se observó en el dominio «ronquidos» con un valor de 1,457, mientras que el dominio «regularidad y duración del sueño» mostro el mayor valor con 1,987.

En las Tablas 3 y 4, se muestra la matriz de componentes, los estadísticos factoriales y el análisis factorial del cuestionario de trastornos de sueño «BEARS». Se determinó el primer ítem de la escala (factor 1), explicaba el $61.4 \%$ de la varianza, para los 5 reactivos evaluados. La prueba KMO indicó moderada adecuación $(0,659)$, con valores estadísticamente significativos (prueba de esfericidad de Bartlett $\chi^{2} / \mathrm{gl}=$ 2690,817; p = 0,001), Tabla 3.

En todos los dominios se obtuvieron saturaciones (h2) superiores a 0,70, y la mayoría de los reactivos mostraron cargas factoriales moderadas en un rango de 0,528 a 0,687. La mayor saturación se presentó en el dominio «problemas a la hora de dormir» con un valor de 0,829 y una carga factorial de 0,687 , mientras que el dominio «regularidad y duración del sueño», mostró menor valor (h2=0,727), carga factorial $(0,528)$, Tabla 4.

Tabla 1.

en niños y adolescentes de Bogotá, D.C. Colombia, con el cuestionario "BEARS" $(\mathrm{n}=8.862)$

\begin{tabular}{|c|c|c|c|c|c|c|}
\hline Sexo & $\mathbf{n}$ & $\begin{array}{c}\text { Problemas a la } \\
\text { hora de dormir } \\
\text { n (\%) }\end{array}$ & $\begin{array}{l}\text { Excesiva } \\
\text { somnolencia } \\
\text { diurna } \\
\text { n (\%) }\end{array}$ & $\begin{array}{l}\text { Despertares } \\
\text { nocturnos } \\
\text { n (\%) }\end{array}$ & $\begin{array}{c}\text { Regularidad y } \\
\text { duración del sueño } \\
\text { n (\%) }\end{array}$ & $\begin{array}{l}\text { Ronquidos } \\
\text { n (\%) }\end{array}$ \\
\hline \multicolumn{7}{|l|}{ Varones } \\
\hline 9 а 9,9 & 277 & 50 (18) & 60 (22) & 61 (22) & 89 (32) & 17 (6) \\
\hline 10 a 10,9 & 498 & $99(20)$ & 97 (19) & $107(21)$ & $140(28)$ & 55 (11) \\
\hline 11 a 11,9 & 480 & 89 (19) & 110 (23) & 94 (20) & $152(32)$ & $35(7)$ \\
\hline 12 а 12,9 & 430 & $96(22)$ & $100(23)$ & 71 (17) & $135(31)$ & $28(7)$ \\
\hline 13 а 13,9 & 437 & $68(16)$ & $111(25)$ & 78 (18) & $131(30)$ & 49 (11) \\
\hline 14 а 14,9 & 477 & 95 (20) & $128(27)$ & $97(20)$ & 155 (32) & $2(0.5)$ \\
\hline 15 a 15,9 & 478 & $82(17)$ & 164 (34) & 81 (17) & 146 (31) & $5(1)$ \\
\hline 16 a 16,9 & 418 & 91 (22) & $133(32)$ & 73 (17) & 121 (29) & $0(0)$ \\
\hline 17 a 17,9 & 290 & $66(23)$ & $101(35)$ & 38 (13) & 85 (29) & $0(0)$ \\
\hline Total & 3785 & $736(18)$ & $1004(22)$ & $700(22)$ & $1154(32)$ & $191(6)$ \\
\hline $\begin{array}{c}\text { Mujeres } \\
9 \text { a } 9,9\end{array}$ & 346 & 750 & 97 (28) & $75(2$ & $99(29)$ & $0(0)$ \\
\hline 10 a 10,9 & 751 & $157(21)$ & 220 (29) & $152(20)$ & 219 (29) & $3(0)$ \\
\hline 11 a 11,9 & 706 & 155 (22) & 214 (30) & 122 (17) & $206(29)$ & $9(1)$ \\
\hline 12 а 12,9 & 591 & $103(17)$ & $180(30)$ & 102 (17) & 198 (34) & $8(1)$ \\
\hline 13 а 13,9 & 566 & 98 (17) & $187(33)$ & 100 (18) & $177(31)$ & $4(1)$ \\
\hline 14 a 14,9 & 704 & $146(21)$ & $198(28)$ & 99 (14) & 259 (37) & $2(0)$ \\
\hline 15 a 15,9 & 580 & 106 (18) & 196 (34) & $128(22)$ & $148(26)$ & $2(0)$ \\
\hline 16 a 16,9 & 504 & $103(20)$ & $167(33)$ & 92 (18) & $133(26)$ & 9 (2) \\
\hline 17 a 17,9 & 329 & 91 (28) & 99 (30) & 78 (24) & 55 (17) & 6 (2) \\
\hline Total & 5077 & $1034(22)$ & $1558(28)$ & $948(22)$ & $1494(28)$ & $43(1)$ \\
\hline
\end{tabular}

\section{Total}

Tabla 2.

Índice de discriminación y coeficiente alfa de Cronbach, si se elimina el dominio del cuestionario de trastornos del sueño "BEARS" ( $\mathrm{n}=8.862)$

\begin{tabular}{lccc}
\hline \multicolumn{1}{c}{ BEARS (dominio) } & $\begin{array}{c}\text { Varianza de la } \\
\text { escala si se } \\
\text { elimina el } \\
\text { elemento }\end{array}$ & $\begin{array}{c}\text { Correlación } \\
\text { ítem-total } \\
\text { corregida }\end{array}$ & $\begin{array}{c}\text { alfa de } \\
\text { Cronbach } \\
\text { si se elimina el } \\
\text { ítem }\end{array}$ \\
\hline 1. Problemas a la hora de dormir & 1,884 & 0,656 & 0,706 \\
2. Excesiva somnolencia diurna & 1,907 & 0,573 & 0,748 \\
3. Despertares nocturnos & 1,921 & 0,629 & 0,720 \\
4. Regularidad y duración del sueño & 1,987 & 0,531 & 0,769 \\
5. Ronquidos & 1,457 & 0,579 & 0,718 \\
\hline
\end{tabular}

Tabla 3.

Matriz de componentes y estadísticos factoriales del cuestionario de trastornos del sueño, BEARS ( $\mathrm{n}=8.862)$

\begin{tabular}{|c|c|c|c|}
\hline Estadístico & Total & Varianza (\%) & Acumulado (\%) \\
\hline \multicolumn{4}{|l|}{ Factor } \\
\hline Factor 1 & 2,456 & 61,408 & 61,408 \\
\hline \multicolumn{4}{|l|}{ Análisis factorial } \\
\hline Índice KMO & 0,659 & & \\
\hline Prueba de Barlett ( $\left.\mathrm{X}^{2}\right)$ & 2690,817 & & \\
\hline Grados de libertad & 6 & & \\
\hline Coeficiente alfa de Cronbach & 0,732 & & \\
\hline Significancia & 0,001 & & \\
\hline \multicolumn{4}{|c|}{$\begin{array}{l}\text { KMO: Índice de adecuación de Kaiser-Meyer-Olkin. Método de extracción: componentes } \\
\text { principales }\end{array}$} \\
\hline \multicolumn{4}{|c|}{$\begin{array}{l}\text { Tabla } 4 . \\
\text { Análisis factorial de trastornos del sueño cuestionario BEARS (n=8.862) }\end{array}$} \\
\hline Ítem & & Saturaciones & Comunalidades \\
\hline 1. Problemas a la hora de dormir & & 0,829 & 0,687 \\
\hline 2. Excesiva somnolencia diurna & & 0,809 & 0,586 \\
\hline 3. Despertares nocturnos & & 0,765 & 0,655 \\
\hline 4. Regularidad y duración del sueño & & 0,727 & 0,528 \\
\hline 5. Ronquidos & & 0,780 & 0,624 \\
\hline
\end{tabular}

Método de extracción: componentes principales. Varianza total: 61,408\%

Tabla 5.

Reproducibilidad del cuestionario de trastornos del sueño BEARS, (n=246) Bep 1, Rep 2, IC95\% (dominio)

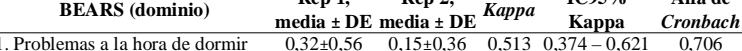
$\begin{array}{lllllll}\text { 1. Problenas a la hora de dormir } & 0,32 \pm, 56 & 0,15 \pm, 36 & 0,513 & 0,374-0,621 & 0,706\end{array}$ $\begin{array}{llllll}\text { 2. Excesiva somnolencia diurna } & 0,42 \pm 1,59 & 0,26 \pm 0,44 & 0,587 & 0,469-0,679 & 0,748\end{array}$ $\begin{array}{lllllll}\text { 3. Despertares nocturnos } & 0,28 \pm 0,56 & 0,15 \pm 0,36 & 0,378 & 0,200-0,516 & 0,720\end{array}$ \begin{tabular}{llllll} 
5. Regularidad y duración del sueño & $0,55 \#, 58$ & $0,33 \pm, 47$ & 0,629 & $0,523-0,711$ & 0,769 \\
5. Ronquidos & $0,51 \#, 52$ & $0,48 \pm, 57$ & 0,529 & $0,499-0,691$ & 0,718 \\
\hline
\end{tabular} Rep: repetición. DE: desviación estándar
En la sub-muestra de escolares ( $\mathrm{n}=246$ ), se presenta la fiabilidad del cuestionario de trastornos de sueño «BEARS», en 7 días de diferencia entre la repetición 1 y 2. Se observa valores aceptables de Kappa ponderado (rango 0,378 y 0,629), y valores individuales aceptables del alfa de Cronbach (todos superiores a 0.70), Tabla 5.

\section{Discusión}

El propósito de este estudio fue determinar la confiabilidad y validez de contenido del cuestionario BEARS en una muestra de niños y adolescentes escolares de 9 a 17 años pertenecientes al estudio FUPRECOL (Ramírez-Vélez et al., 2015); -instrumento desarrollado para evaluar los TS desde etapas tempranas de la vida-. Como principales resultados de este estudio, se muestran que el cuestionario «BEARS», posee propiedades psicométricas adecuadas y suficientes, tanto en escala global, como en cada una de sus dimensiones en términos de fiabilidad y validez (García et al., 2009).

Respecto a la consistencia interna, el cuestionario BEARS alcanzó un alfa =0,732, (rango 0,706 y 0,769), confirmando la existencia de una escala fiable, de acuerdo a lo sugerido por Oviedo \& Campo (2005), quienes describieron que valores superiores a 0,70 , revelan una adecuada consistencia interna para un instrumento por auto-reporte. Al comparar los resultados del presente estudio de validación, con otros trabajos que aplicaron en población escolar el cuestionario «CSHQ-BEARS», encontramos algunas diferencias con el informe alemán (Waumans etal., 2010), aplicado en 2.385 niños. Por ejemplo, estos autores reportaron menores valores para el coeficiente alfa (entre 0,47 a 0,68). Este mismo hallazgo, fue reportado en la versión Norteamericana (Owens et al., 2000), ya que los valores de alfa oscilaron entre 0,36 y 0,70 . Por su parte, la versión Española mostró valores muy similares a descrito en este reporte con rangos 0,56 y 0,81 (Lucas-de la Cruz et al., 2016), resultado similar al descrito en la versión Persa (Fallahzadeh et al., 2015), con un alfa de 0,80. Finalmente, la versión China (Li et al., 2007), aplicada en 20.457 infantes, mostró un alfa de Cronbach de 0,73 (rango entre 0,42-0,89). Otro trabajo español, realizado por Bastida-Pozuelo et al. (2016), se encontró un coeficiente alfa de 0,73.

Estas divergencias pueden explicarse en parte a que las sub-escalas originales de la «CSHQ-BEARS» se basan en trastornos clínicos (Waumans et al., 2010; Owens et al., 2000), aspecto que ha demostrado alteraciones con la higiene del sueño. Otra posible explicación, es la edad de la población evaluada, el estrato socioeconómico, el apoyo familiar, y las variaciones culturales, tales como compartir habitación con los padres, -lo cual es frecuente en las familias Orientales y Persas-, o aspectos políticos de un tener un hijo por pareja en el medio oriente, podría explicar las diferencias encontradas en la higiene y patrón de sueño (Liu et al., 2005).

Complementario a lo descrito anteriormente, la validez de contenido resultó adecuada para todos los reactivos y dominios del cuestionario «BEARS», con una concordancia promedio de 0,629 , valor que muestra un aceptable grado de acuerdo, según lo descrito por Viera \& Garrett (2005). Otros estudios que han evaluado la concordancia del "CHSQ- BEARS», muestran resultados similares (Waumans et al., 2010; Owens et al., 2000; Lucas-de la Cruz et al., 2016; Fallahzadeh et al., 2015; Li et al., 2015), a pesar de haber utilizado el coeficiente de correlación intraclase. En esta misma línea, el AFE mostró una varianza explicada de $61,40 \%$, aunque se ha descrito que los valores de la varianza explicada deben estar por encima de 80\% (Ochoa-Meza et al., 2014). No obstante, este porcentaje no se ha encontrado en estudios previos, por lo que valores superiores a 60\%( Ochoa-Meza et al., 2014), son aceptables para que el número de factores validen un cuestionario.

El método de extracción empleado en este estudio fue el análisis de componentes principales, técnica estadística respaldada por el índice de adecuación de Kaiser-Meyer-Olkin o KMO, el cual mostró en este estudio una buena adecuación muestral de los datos al modelo de análisis e indicó la proporción de la varianza que poseen en común las variables estimadas (Fernández \& Díaz 2016). El estadístico KMO encontrado para este estudio fue de 0,659 , lo que muestra el ajuste de las 
variables sustentadas por la prueba de esfericidad de Bartlett $\left(\chi^{2}=2690,817 ; p=0,001\right)$. En adición, los resultados de este estudio muestran comunalidad entre los 5 factores, con valores mayores a 0,528. Estos datos coinciden con la teoría psicométrica (Nunnany 1978), dondese afirma que cada factor deberá contener variables que se correlacionen alta y exclusivamente con ese factor, por encima de 0,50. Estudios que han evaluado AFE en la escala original de 22 reactivos, muestran una estructura de 10 factores en España (Lucas-de la Cruz et al., 2016); ocho factores en la versión en alemán (Waumans et al., 2010) y Persa (Fallahzadeh et al., 2015), mientras que la versión China (Li et al., 2007), mostró solo tres factores (con una varianza explicada del 58,63\%). Finalmente, en la versión Norteamericana (Owens et al., 2000) no se reportó ningún criterio de validez.

\section{Limitaciones}

A pesar de que el instrumento presenta limitaciones inherentes a todos los instrumentos de auto-reporte, como los prejuicios sociales y la selección por conveniencia de la muestra poblacional, se ha demostrado que el cuestionario BEARS, es válido y fiable para la evaluación de los TS en escolares de 9 a 17 años. Otra limitación es la inherente a su carácter transversal, adicional a la existencia del sesgo de selección, limitando la participación de escolares de otras áreas geográficas de Bogotá, Colombia; así como el rango de edad de los participantes (9a 17 años) incluidos en este trabajo. Tampoco fue incluido las horas de sueño entre semana o fines de semana, las prácticas alimentarias, los niveles de actividad/condición física, el estado metabólico, la composición corporal y otras variables de la salud física como la morbilidad sentida, todas estas, descritas como factores asociados con la higiene del sueño. No obstante, las limitaciones descritas en este trabajo no comprometen los resultados obtenidos en la población estudiada y posteriores estudios de la cohorte FUPRECOL, se encuentran explorando estas relaciones.

Como fortaleza de este trabajo, cabe mencionar la utilización del cuestionario BEARS que, aunque se considere que puede generar un sub-registro en las dimensiones exploradas, se considera que cuenta con las propiedades psicométricas de validezy de confiabilidad suficientes para ser aplicado como instrumento adecuado para el tamizaje de conductas relacionas con TS en poblaciones escolarizadas. Contar con una muestra poblacional numerosa de ambos sexos, ofrece nuevas perspectivas acerca del desarrollo y el comportamiento de los patrones de sueño y sus implicaciones en salud en niños y adolescentes colombianos. Se hace necesario promover programas de prevención en las alteraciones del sueño como una conducta no saludable que afecta la salud y el desempeño del niño escolarizado.

A pesar de ello, futuras investigaciones con muestras representativas procedentes de otros contextos regionales de Colombia, deberán estimar la confiabilidad test-retest y la validez confirmatoria de los constructos que permitan encontrar mayores evidencias de distintas fuentes de validez. Destacamos que, a la fecha, este es uno de los primeros estudios de validación realizados con población escolar colombiana, que describe el marco conceptual a partir del cual se pueda aplicar el cuestionario BEARS.

\section{Conclusiones}

Los resultados de este estudio aportan evidencias de validez suficiente de la versión colombiana del instrumento BEARS para la evaluación de los TS en escolares de 9 a 17 años. Estudios posteriores deberán continuar con el proceso de aportar evidencias de validez confirmatoria y validez divergente en otras latitudes de Bogotá y de Colombia.

\section{Aplicaciones Prácticas}

Se ha descrito una relación entre desórdenes del sueño y rendimiento académico. Además, una adecuada calidad del sueño se asocia a mejores funciones ejecutivas mentales. Con este cuestionario, se podrían promover programas de prevención en la escuela que busquen la higiene del sueño como un indicador de salud asociado al rendimiento académico. Igualmente es de utilidad para todos los profesores que quieran evaluar y realizar un seguimiento de la evolución de la calidad de sueño de sus estudiantes, así como para su uso en encuestas poblacionales.

\section{Conflicto de intereses}

Los autores del estudio declaran no tener conflicto de interés.

\section{Financiación}

El presente trabajo forma parte del Proyecto FUPRECOL (Asociación de la Fuerza Prensil con Manifestaciones Tempranas de Riesgo Cardiovascular en Niños y Adolescentes Colombianos) financiado por el Departamento Administrativo de Ciencia, Tecnología e Innovación Colciencias, Contrato No 122265743978.

\section{Agradecimientos}

Los autores agradecen la colaboración de escolares, profesores y personal administrativo de las instituciones educativas que se involucraron activamente en este estudio, así como también a las autoridades de la Secretaría de Educación Distrital de Bogotá que aprobaron la realización de esta investigación.

\section{Referencias}

Alamian, A., Wang, L., Hall, A., Pitts, M., \& Ikekwere, J. (2016). Infant sleep problems and childhood overweight: Effects of three definitions of sleep problems. Prev Med Rep, 25(4), 436-438.

Anderson, B., Storfer-Isser, A., Taylor, H., Rosen, C., \& Redline, S. (2009). Associations of executive function with sleepiness and sleep duration in adolescents. Pediatrics, 123(4), e701-707.

Aristizábal, J., Restrepo, M., \& Estrada, A. (2007). Body composition assessment by anthropometry and bioelectrical impedance. Biomédica, 27(2), 216-224.

Ato, M., López-García, J. J., \& Benavente, A. (2013). A classification system for research designs in psychology. Anales de Psicologia, 29(3), 1038-1059

Bastida-Pozuelo, M., \& Sánchez-Ortuño, M. (2016). Preliminary analysis of the concurrent validity of the Spanish translation of the BEARS sleep screening tool for children. J Psychiatr Ment Health Nurs, 23(8), 513-520.

Chaput, J., Lambert, M., Gray-Donald, K., McGrath, J., Tremblay, M., \& O’Loughlin, J. (2011). Short sleep duration is independently associated with overweight and obesity in Quebec children. Can J Public Health, 102(5), 369-374.

Choi, K., Lee, J., Park, H., Baik, S., Choi, D., \& Kim, S. (2008). Relationship between sleep duration and the metabolic syndrome: Korean National Health and Nutrition Survey 2001. Int J Obes (Lond), 32(7), 1091-1097.

Fallahzadeh, H., Etesam, F., Sadat., \& Asgarian, F. (2015). Validity and reliability related to the Persian version of the Children's Sleep Habits Questionnaire. Sleep and Biological Rhythms. 2015; 13(3), 271-278.

Fernández, P., \& Díaz, P. (2016). [Exploratory factorial analysis and factorial complexity: Beyond rotations. Enferm Clin, 11308621(16), 30059-3066.

Garaulet, M., Ortega, F., Ruiz, J., Rey-López, J., Béghin, L., \& Manios, Y. (2011). Short sleep duration is associated with increased obesity markers in European adolescents: effect of physical activity and dietary habits. The HELENA study. Int J Obes (Lond), 35(10), 1308-1017.

García, M., Rodríguez, F., \& Carmona, L. (2009). Validación de cuestionarios. Reumatología Clinica, 5(4), 171-174.

García-Jiménez, M., Salcedo-Aguilar, F., Rodríguez-Almonacid, F., 
Redondo-Martínez, M., Monterde-Aznar, M \& Marcos-Navarro, A. (2004). Prevalencia de los Trastornos del Sueño en Adolescentes en Cuenca, España. Rev Nerol, 39(1), 18-24.

Giannotti, F., Cortesi, F., Sebastiani, T., \& Ottaviano, S. (2002). Circadian preference, sleep and daytime behaviour in adolescence. J Sleep Res, 11(3), 191-199.

Gruber, R., Somerville, G., Enros, P., Paquin, S., Kestler, M., \& GilliesPoitras, E. (2014). Sleep efficiency (but not sleep duration) of healthy school-age children is associated with grades in math and languages. Sleep Med, 15(12): 1517-1525.

Ipsiroglu, O., Fatemi, A., Werner, I., Paditz, E., \& Schwarz, B. (2002). Self-reported organic and nonorganic sleep problems in schoolchildren aged 11 to 15 years in Vienna. J Adolesc Health, 31(5), 436-442.

Kong, A., Wing, Y., Choi, K., Li, A., Ko, G., \& Ma, R. (2011). Associations of sleep duration with obesity and serum lipid profile in children and adolescents. Sleep Med, 12(7), 659-665.

Li, S., Jin, X., Shen, X., Wu, S., Jiang, F., \& Yan, C. (2007). Development and psychometric properties of the Chinese version of Children's Sleep Habits Questionnaire. Zhonghua Er Ke Za Zhi, 45(3), 176180.

Liu, X., Liu, L., Owens, J., \& Kaplan, D. (2005). Sleep patterns and Sleep problems among schoolchildren in the United States and China. Pediatrics, 115(1 Supl 1), 241-219.

Liu, X., Sun, Z., Uchiyama, M., Shibui, K., Kim, K., \& Okawa, M. (2000). Prevalence and correlates of sleep problems in Chinese schoolchildren. Sleep, 23(8), 1053-1062.

Lucas-de la Cruz, L., Álvarez-Bueno, C., Arias-Palencia, N., \& Notario-Pacheco, B. (2016). Reliability and validity of the Spanish version of the Children's Sleep Habits Questionnaire (CSHQ-SP) in school-age children. Child Care Health Dev 42(5), 675-682.

Martins, J.C., Honorio, S., Costa, A.M., Batista, M., \& Cardoso, J. (2014). Longitudinal study of physical fitness levels, BMI and childhood obesity in school context. J. Hum. Sport Exerc. 9(2), 645-655.

Mindell, J., \& Owens, J. (2003). Sleep problems in a pediatric practice: clinical issues for the pediatric nurse practitioner. J Pediatr Health Care, 17(6), 324-331.

Miró, E., Cano-Lozano, MDC., \& Buela-Casal, G. (2005). Sueño y calidad de vida. Rev. Colomb. Psicol, 14, 11-27.

Nunnany, J. (1978). Psychometric theory. 2nd ed. New York: McGraw
Hill.

Ochoa-Meza, G., Sierra, J., Pérez-Rodrigo, C., \& Aranceta-Bartrina, J. (2014). Validity of Pro Children Project questionnaire for assessing psychosocial factors of fruit and vegetable intake in Mexico. Salud Publica Mex, 56(2), 165-179.

Oviedo, H., \& Campo, A. (2005). Aproximación al uso del coeficiente alfa de Cronbach. Rev Col Psiq, 24(4),572-580.

Owens, J., \& Dalzell, V. (2005). Use of the 'BEARS' sleep screening tool in a pediatric residents continuity clinic: a pilot study. Sleep Medicine, 6(1), 63-69.

Owens, J., Spirito, A., \& McGuinn. M. (2000). The Children’s Sleep Habits Questionnaire(CSHQ): Psychometric properties of a survey instrument for schoolaged children. Sleep, 23(8), 1043-1051.

Paavonen, E., Aronen, E., Moilanen, I., Piha, J., Räsänen, E., \& Tamminen, T. (2000). Sleep problems of school-aged children: a complementary view. Acta Paediatr, 89(2), 223-228.

Pérez-Moreno, P., Calzada-Álvarez, N., Rovira-Guardiola, J., \& TorricoLinares, E. (2012). Estructura factorial del test ASSIST: aplicación del análisis factorial exploratorio y confirmatorio. Trastornos Adictivos, 12(2), 44-49.

Ramírez-Vélez, R., Rodríguez-Bezerra, D., Correa-Bautista, J. Izquierdo, M., \& Lobelo F. (2015). Reliabity of Health-Related Physical Fitness Test among Colombian Children and Adolescents: The FUPRECOL Study. PLos ONE, (10), e0140875.

Ravikiran, S., Kumar, P., \& Latha, K. (2001). Sleep problems in preschool and school aged rural Indian children. Indian Pediatr, 48(3), 221223.

Salsberg, J., Soultana, M., Bengoechea, E.,G., Macaulay, A.,C. Moore, S. (2015). Engaging Community Stakeholders for School-Based Physical Activity Intervention. RETOS , 28, 104-109.

Smith, S., \& Trinder, J. (2001). Detecting insomnia: comparison of four selfreport measures of sleep in a young adult population. J Sleep Res, 10(3), 229-235.

Viera, A., \& Garrett, J. (2005). Understanding interobserver agreement: the kappa statistic. Fam Med, 37(5), 360-363.

Waumans, R., Terwee, C., Van den Berg, G., Knol, D., Van, LR., \& Gemke, R. (2010). Sleep and sleep disturbance in children: Reliability and validity of the Dutch version of the Child Sleep Habits Questionnaire. Sleep, 33(6), 841-846.

Webb, W., Bonnet, M., \& Blume, G. (1976). A post-sleep inventory. Percept Mot Skills, 43, 987-993.

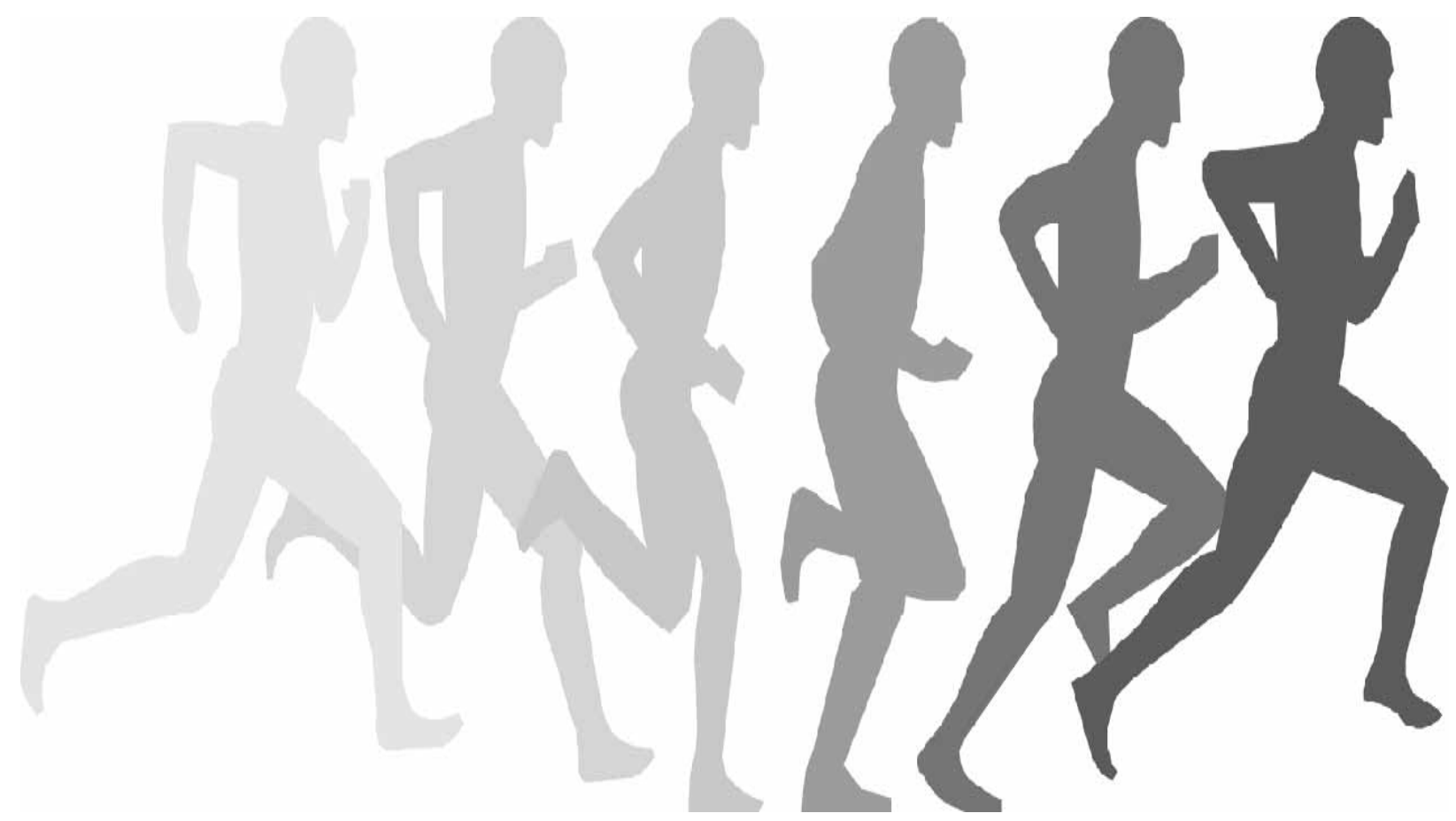

